\title{
Automatic Analysis System of Network Incidents
}

\author{
J. Javier Ferro ${ }^{1}$, Hugo Gurendez ${ }^{2}$ David MacDonald ${ }^{2}$ Javier Amantegui $^{2}$ and Cesáreo Fernández ${ }^{3}$ \\ ${ }^{1}$ Departament of telecomunications and telecontrol \\ Iberdrola ingeniería y construcción S.AU. \\ Ribera de Axpe, 5, 48950 Erandio, Spain \\ Phone:+0034 946 019214, e-mail: jferro@iberdrola.es \\ ${ }^{2}$ Departament of proteccions and tecnical assitance \\ Iberdrola Distribución S.A. \\ Avda San Adrian, 48, 48003 Bilbao, Spain \\ hgurendez@iberdrola.es \\ dmacdonald@iberdrola.es \\ javier.amantegui@iberdrola.es \\ ${ }^{3}$ Institute for Research in Technology \\ Universidad Pontificia de Comillas. \\ Alberto Aguilera , 5, 28015, Madrid, Spain \\ cesar@iit.upcomillas.es
}

\begin{abstract}
This article presents the automated processing system of network incidents, as developed and implemented by Iberdrola SA. The SASIRE system was developed due to the need by power companies, of the early identification and resolution of incidents occurring in the high and medium voltage power grid. SASIRE detects, processes and analyses system disturbances and automatically generates network incident reports. By acquiring data from the intelligent electronic devices (IEDs) installed on the network, the information from the network control systems and the knowledge of the incident analysts, it was possible to create automatic incident reports, improved and enhanced sharing of information between the various internal stakeholders and the extraction of knowledge from expert analysts. Throughout the development process, the need for new data, features and functionality in protection and control plant was discovered.
\end{abstract}

\section{Key words}

Relaying Power Systems, Power Systems Incident, Automatic Analisys.

\section{Introduction}

One of the key challenges of electrical utilities [1] is the detection and resolution of incidents that occur in the Electricity Distribution Network. This is due to the importance of quality of service, which is seen as a critical factor for both operators and consumers. Therefore, it is necessary to analyze in detail all system disturbances. If not identified and addressed early, they can cause considerable damage, increasing operating costs, even causing serious incidents that can lead, first in sanctions by regulatory or administrative authorities, and the other in the declining quality of service and thereby deteriorating consumer confidence.
For example, those serious blackouts in Europe and USA in recent years, gave rise to significant economic losses and loss of consumer confidence in the reliability of electricity supply.

The article presented here reflects the strategy to improve efficiency in the transmission and distribution of electric power, as well as to increase the quality and reliability of the service offered. In section II and III, the evolution of the energy model will be presented, as will the specific environment in which the system has been implemented for automatic processing of network incidents. Section IV and $\mathrm{V}$ will present the main objectives of the project and how they were achieved. Subsequently, the final conclusions of the project are presented in Section VI. And finally Section VII explains possible future lines of action.

\section{Evolution of the energy model}

The Spanish electricity distribution network was designed for needs and requirements very different from those that exist today. The operating model of the current grid, from generation, transmission, distribution and consumption, is being transformed to a distributed energy model [2], which allows the easy connection and disconnection of both points of generation and consumption. This distributed model will facilitate future integration into the network of local renewable microgeneration, it will allow the storage of electricity and enable the connection of electric vehicles.

The management of such networks will promote the reduction of transmission losses, since the points of generation and consumption are not as far apart. In Fig 1 the current energy model is shown along with its expected 
future development, and can be seen on the integration of distributed generation and energy storage systems.

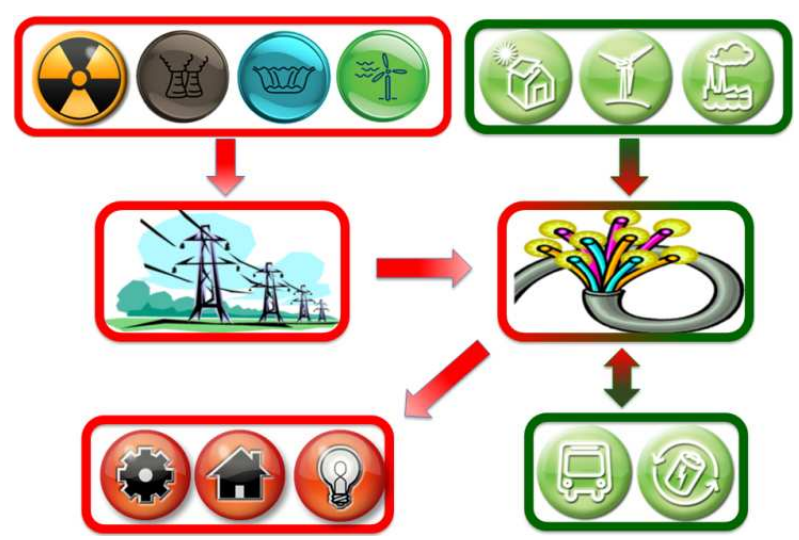

Fig. 1. Current Energy Model and its transformation.

This fact, together with the expected increase in electricity demand nationally and internationally, pushing electrical systems towards their physical limits, could have the effect of significantly reducing the reliability of the operating environment, increasing the likelihood of incidents occurring with an unpredictable impact on the power network.

\section{Environment}

Traditionally communications with the substations were the exclusive privilege of the SCADA / RTU, so that network operators could supervise the behavior of the network, and its main purpose was to operate switches and circuit breakers [3].

Later, with the emergence of communicable IEDs in substations, the main objectives were: the protection of network elements, ease of installation, ease of adjustment and maintenance thereof. This, together with the different demands of times, details and data required in protection and control, has contributed to the development of new digital equipment which has evolved in a separate form. The continuing process of development has led to the emergence of a variety of control, measurement and protection equipment, which has added to the lack of standardization in these fields and the variety of manufacturers within them. Consequently this has led to the emergence of a multitude of different communication protocols whilst it is difficult to jointly install different equipment in one substation constituting an integrated system. It is becoming increasingly clear of the inability of existing equipment to meet current and future requirements, forcing the need to adapt or update. Communicable electronic devices represent a significant advance in the integration concept, aimed at optimizing the management of power systems, because they provide large amounts of useful and necessary data, not only to the IEDs themselves, but also to: maintenance teams, facility managers and managers of new customer services. Within utilities, most of the divisions want, somehow, to access data from the substation, not exactly in real time, but directly.

The Integrated Incident Analysis tool (SINAI) [4], provides the protections department of IBERDROLA, SA a software tool to facilitate some tasks they perform. These tasks include the following:
- A full Inventory of relays and control systems installed on the network.

- Acquisition and storage of standardized data from the IEDs.

- Presentation of the data needed to perform the analysis of incidents, by qualified personnel.

SINAI acquires the remote SCADA information to automatically update the database of the elements of the network, and alarms to detect relay operations in the network. This generates automatic calls to the IEDs affected, acquiring, using the appropriate protocol, the data recorded by them. This data is standardized, classified and stored for later use in the analysis of incident, by skilled users, as shown in Fig 2.

Therefore Sinai becomes a crucial step for automatic analysis of incidents, as it offers the management of communications with remote IEDs and a powerful standardized database, managing all the data involved in a given incident, from IEDs (events, oscilloscope, fault reports, etc.). Sinai has established relationships with internal databases of the company, such as GAMAD the maintenance and asset management tool.

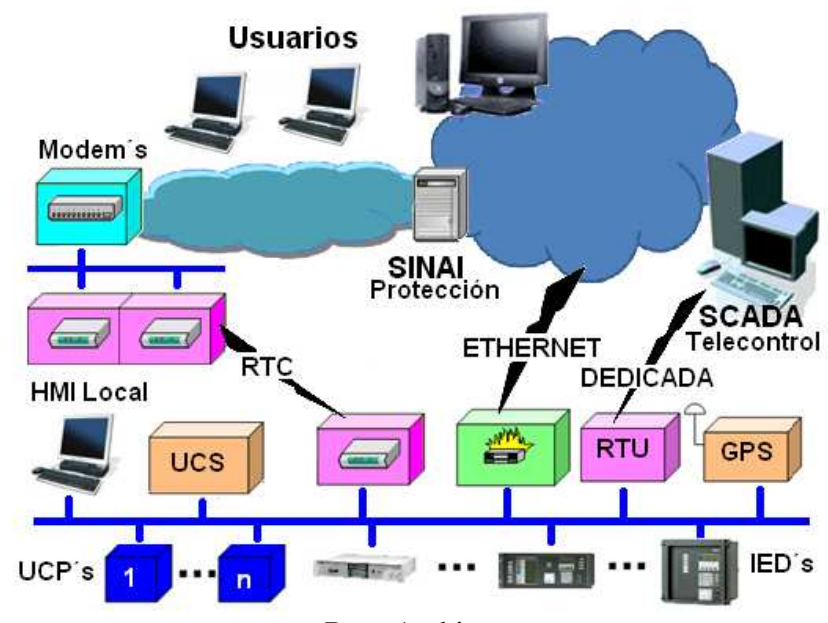

Fig. 2. Base Architecture.

It may be the case that the Sinai system does not have all the data involved for a given incident, at the time of the analysis, because it is an asynchronous system, which depends on the quality of communications with IEDs. Today, Sinai, has an effectiveness of $83 \%$ correct and complete communication, of the nearly 14,000 installed equipment, with annual growth of 1600 teams.

IEDs are increasingly offering access to more data and functions and feature a performance of high communicability [3], but have limited storage capacity and data in case of avalanche, usually overflowing, which presents a loss of data.

In this environment, as you can tell from the above, we find the following difficulties:

- Lack of standardization in the data: because manufacturers offer similar but not identical data on each relay models, and they also provide data or unique features on their computers to keep their product.

- Lack of synchronization: because synchronization systems IEDs. Data from IEDs in the same substation, and more often between different substations may not be synchronized. 
- Lack of topological relations: due to a lack of connectivity between the IEDs.

- Communications Delay: due to limited IED's communications with the absence of dedicated communications, there is a time period in which the database is not updated with the actual data.

- Data loss: due to storage limitations in the absence of communications IED's and perpetual avalanche if the IED itself removes older data to store the most recent.

\section{Objetives}

The draft analysis of events and oscillographic records of digital protection during incidents have the following objectives:

a) To automatically assess the performance of network protections radial MT and AT.

b) To identify risk and hidden problems in the distribution network.

c) To provide useful information for maintenance and asset management lines. EHV.

d) To facilitate the analysis of protections in HV and

Featured in this article is the basic design of a system for generating and analyzing data for automatic incident detection on high and medium voltage networks, standardising a single system that is appropriate for all utilities, improving data exchange between the different systems [5] to control the network and also undertaking the design and development of new features and capabilities for new protection and control equipment for the generation of new signals.

This system will allow for rapid detection of incidents, monitoring and automated analysis of network incidents, both above and below $45 \mathrm{kV}$, and for the implementation of predictive maintenance based on statistical data, the detection of recurring faults in the network and the estimation of the useful life of plant.

It should be noted that up until now, incidents have not been systematically analyzed in networks under $45 \mathrm{kV}$, because of the unavailability of time or resources necessary for this.

Automatic analysis of network incidents responds to the need for electric utilities to communicate remotely and in parallel with the SCADA / RTU infrastructure, with smart devices installed in electrical substations, so that it can access data stored in them for further processing and analysis.

It is now becoming clear just how important proper data management is. And with proper treatment of the data acquired from the IED's annex systems, one can determine whether to continue maintaining plant or to replace it. Correct decision making in this area has the ultimate result of improving the quality of supply whilst minimizing the ownership cost of the assets to the utility. This will facilitate electrical companies to progress in the process of new product adaptation through the incorporation of all those elements that eliminate the need of the existence of their own solution, obtain the independence of the components and suppliers, provide the teams that use and commercialise the elements of connectivity based on internet technologies and to be ready for the changes ahead.

\section{Automatic analysis process}

To optimize the automated analysis of incidents from event logs in Sinai, this analysis has been structured in three levels:

Level 1 (protection): Automatic analysis from the incident only, data from the protection relay.

Level 2 (substation): Automatic analysis of the incident from the information generated by multiple relays incidents of the same substation and with the same time intervals.

Level 3 (network): Automatic analysis of the incident from the information generated from several incidents of several substations connections protections and with the same time intervals.

Network alarms are requested by the SINAI system, to communicate with the IEDs involved in the incident and acquires the data recorded the incident. Once data protection events are categorized and stored. The analysis module performs automatic analysis of event data and automatically generates the incident level 1 if necessary. The incident is determined by the date of occurrence and cause element, as seen in Fig 3, and is divided into a sequence of events, reviews, faults and failures. The data model of the incident is identical to that used in the analysis manual and on top of this, the same software (SINAI) is used. Therefore additional costs of adoption are avoided.

The data originators or creators of a problem are an ordered series of events determined within intervals of time, which meets set requirements. This module examines the event $\log$ of a relay, deciding whether it constitutes one operation or a sequence of operations.

Therefore identifying the element causing the event log and calculating the type of action and the duration of each of the operations, assesses the operating behaviour to: the model of ideal relay, the circuit breaker, the recloser and the protection relay. Then it determines the type of action (for example trip without reclose), valuation (for example a fault in the closing operation) and other relevant details of the complete operation.

The process continues once the incident report is created, verifying that the report is not related to another existing incident report, checking times and topological network connections [6]. If there are no related incidents the process is finished and the original event logs, analysis of the oscilograph, fault analysis, created comments and fault diagnosis are presented in the incident report.

Otherwise, both incidents are merged together using the registered time of the oldest, which would be the principal record. Due to the merger of the incident reports, some information which may be subject to change is recalculated, for example; faults, comments, final diagnosis, etc. All the oscillographs and event logs of the incident are moved to the report of the principal incident. 


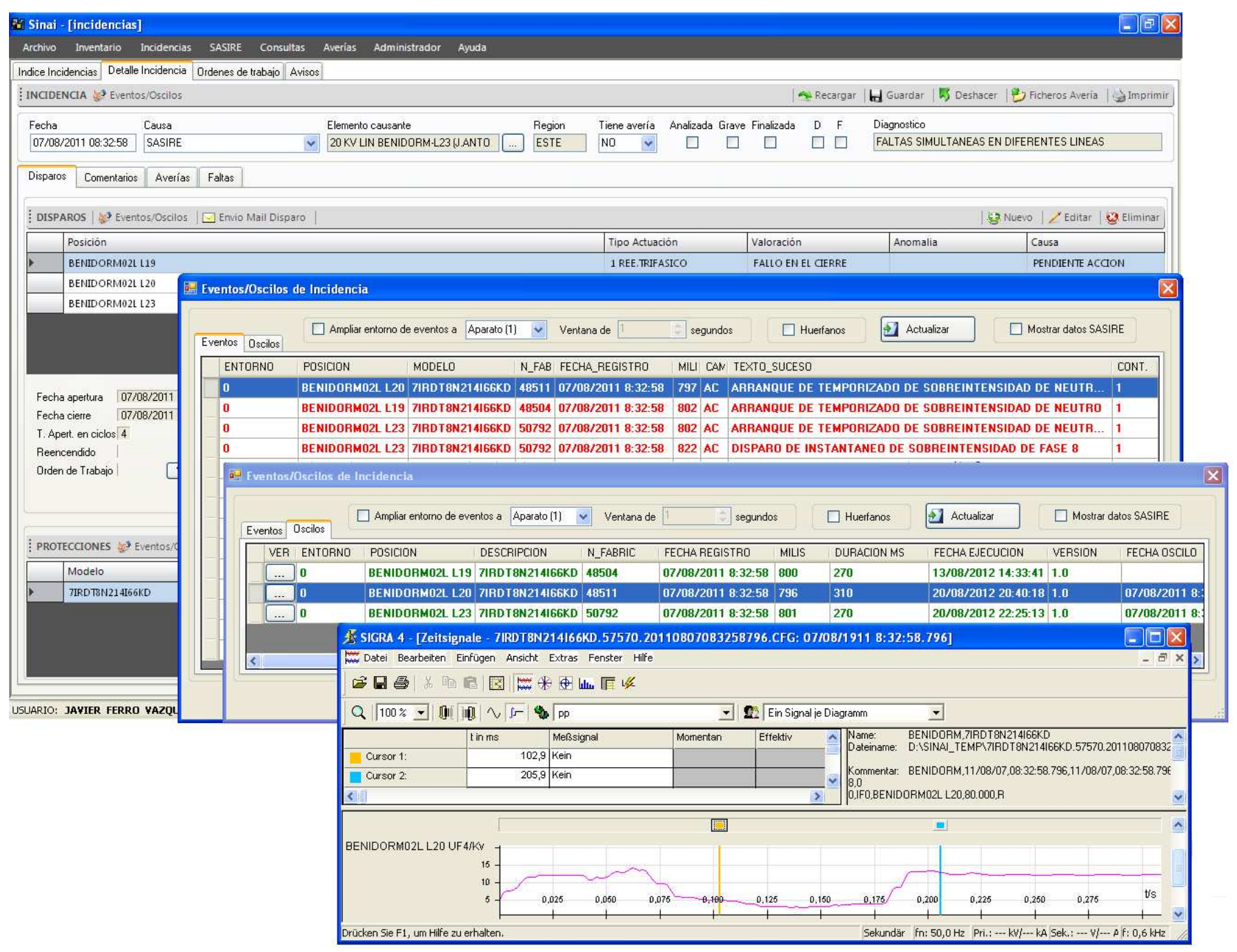

Fig. 3. The user window where the incident details are shown as well as the event log and related oscillographs.

\section{Conclusion}

The benefits derived from the implementation of this system will be seen by all stakeholders involved in the value chain of the power sector; from the equipment manufacturer, contracting companies, power ulities all the way to the client end.

With the implementation of the system, exchange of information between the different actors involved is increased and improved, through the standardizing of data acquired from IED's. This has revealed new necessities in Protection and Control equipment, new data needs, capabilities and functionalities. Consequently, offering manufacturers new lines of development for future equipment.

The process of automatic creation of incident reports has been achieved to; promote knowledge management of the organization [7], significantly reduce analysis time and increase the capability to analyze incidents in the entire network of medium, high and very high voltage levels, minimizing risk to the utility.

The automatic incident analysis system, SASIRE, offers the possibility of using symptoms to enable proactive action to resolve certain abnormalities before they have a serious effect on the network.
Moreover, based on the information generated and what actually happens in the network, the system will act to decide priorities and frequencies of maintenance of network elements and help determine the lifetime of the equipment installed. It also provides the possibility as a potential tool for identifying hidden problems in the network before they can have an impact on the protection system and therefore on the network.

With all this, it can be said that SASIRE, will become an essential tool for businesses involved in the generation, transmission and distribution of electricity.

And its implementation will be essential in the organizations involved in the management of the assets of the companies. By providing a solid foundation in optimizing the quality and security of supply, SASIRE will help small consumers as much as large ones, and supplier companies may acquire a significant competitive advantage.

\section{Future lines}

The scope for future work in SASIRE includes two important points that should be developed. 
The first point is the need to improve the algorithms to extract the information contained in oscillographs, which can open the door for more accurate and higher quality automatic analysis of incidents.

And the second point is the design of a software tool that allows the analyst, based on high-level basic functions, access to the complex relationships between data and standardized information and generation of new information relevant to the organization. This tool should have as a main objective the implementation of algorithms for the analysis of incidents and thereby convert the implicit knowledge of the analyst to explicit knowledge.

Other lines of work not less important which are currently being developed to improve the efficiency of the scheme, are: improving the quality of communications, synchronize data, automating existing topological relations and optimizing the method of standardizing of the data acquired from IEDs.

\section{References}

[1] Normas técnicas y de seguridad de las instalaciones eléctricas, Ley 54/1997 articulo 51, del sector eléctrico.

[2] Jeremy Rifkin, La civilización empática, Paidos Iberica, 2010.

[3] Automatización de Subestaciones, Luc HOSSENLOPPGEC ALSTOHOM T\&D P\&C.

[4] J. Martinez, "Experience with SINAI an integrated incident analysis sytem", Electricity Distribution, Part1: Contributions CIRED. 14th International Conference and Exhibition on (IEE Conf. Publ. No. 438) Vol4, 1997, pags 15/1 - 15/4 vol.4.

[5] Mercedes García Parra, "Los elementos integrantes de la nueva riqueza de la empresa", Intangible capital: $n^{\circ} 1$, Julio 2004.

[6] M. Kezunovic, "Monitoring of Power System Topology in Real-Time," Symposium on Electric Power Systems Reliability, Hawaii, January 2006.

[7] Ikujiro Nonaka and Hirotaka Takeuchi. "The knowledge creating company: How japanese companies create the dynamics of innovation”. Oxford University Press, 1995. 